\title{
Ensayo sobre la categoría de sexo y la perspectiva de género
}

\section{Essay on sex category and gender perspective}

Nolver Guevara Caicedo

Semillero Eureka. Departamento de Humanidades, Facultad de Teología, Filosofía y Humanidades. Fundación Universitaria Católica Lumen Gentium, Cali, Valle del Cauca-Colombia.

Correo electrónico: nguevara@unicatolica.edu.co
Las palabras tienen vida propia que se escapa a cualquier encasillamiento de diccionario, y que los conceptos dibujados con esas palabras son igualmente sujetos de una historia que queda traicionada al ofrecer una imagen fija. (Reinhart, 2004)

\section{Resumen}

Este artículo es un ejercicio de revisión bibliográfica, desde la lectura de algunas feministas latinoamericanas que han pensado el problema de género. El objetivo de este estudio fue aclarar el significado de los términos" perspectiva de género", "género" $y^{\prime \prime}$ sexo"; como también aportar de algún modo, una mirada más tolerante a las dinámicas de las sociedades pluriculturales. El texto inicia hablando de perspectiva de género; luego, de categoría de género y clausura explicando los conceptos sexo y generoelectos. Al final, se llega a la conclusión de los estudios de género, con la aclaración de la categoría de sexo y género, han propiciado, en las últimas décadas, cambiar la mirada hacia la mujer y hacia los otros géneros.

Palabras clave: perspectiva de género, género, sexo y generoelectos.

\begin{abstract}
This article is an exercise of literature review, since the reading of some Latin American feminists who have thought about the problem of gender. The objective of this study was clarify the meaning of the terms "gender perspective", "gender" and "sex"; as well, contribute in some way, a more tolerant look to the dynamics of the pluricultural societies. The text begins speaking of "gender perspective"; then, of "gender category" and closure explaining the con-
\end{abstract}


cepts "sex" and "gender elected". At the end, arrives to the conclusion of the gender studies, with the clarification of the category of "sex" and "gender", have propitiated, in the last decades, change the look toward the woman and the other genders.

Keywords: gender perspective, gender, sex and generoelectos.

\section{Introducción}

La cultura, en la cual viven los individuos, se construye a partir del "sexo", de ciertos comportamientos, actitudes y prácticas que marcan la distinción social entre lo que significa ser hombre y ser mujer. A medida que crecen los individuos, la sociedad refuerza el rol que debe asumir cada individuo; esto implica"formas" de masculinidad y feminidad. Por supuesto, la biología dota al ser humano de rasgos físicos y anatómicos que marcan la pauta de la diferenciación de los personas, pero es la sociedad la encargada de reforzar las formas de ser. Consecuencia de tal situación es la legitimización de la supremacía de un género sobre otro, lo cual causa desagravios e humillaciones y la imposición de una ideología heterosexual. Sin embargo, las dinámicas sociales, en las sociedades modernas, han demostrado que los individuos irrumpen el paradigma y luchan por emanciparse de esta ideología. La comunidad LGTBI es un ejemplo de la expresión de la diversidad de géneros que luchan por ser reconocidos y aceptados.

Los términos "sexo"y "género" se usan, sin reparos, en las conversaciones cotidianas, como si fueran sinónimos, sin percatarse de la diferencia. Actualmente, se piensa que el género masculino caracteriza al hombre y el género femenino, a la mujer. Ante las preguntas: ¿cuál es tu sexo?, la respuesta es hombre o mujer; ¿cuál es tu género?, la respuesta es masculino o femenino. Entonces, ¿cuál es la causa de la ambigüedad del término? ¿Cuáles son las razones subyacentes en las expresiones? ¿Por qué el concepto de género se ha convertido en una categoría revolucionaria para desentrañar la dominación masculina? En últimas, qué ¿qué significa sexo y género? Estas preguntas acompañan las líneas siguientes; de hecho, son necesarias para comprender la importancia del uso del concepto género en la perspectiva de género.

Para Serret Bravo (2008), los aportes de las feministas de mayor relevancia, en la segunda mitad del siglo XX, están en manos de Simone Beauvoir, Cherry Ortner y Gayle Rubin, quienes postulan el sexo y género, como la fuente principal de discriminación, desvalorización, humillación y dominación masculina sobre las mujeres. Más que la negación de los derechos económicos, sociales y políticos, reinvidicados por las mujeres desde el siglo XVIII. Simone de Beauvoir, novelista, filósofa y activista francesa, influenciada, de manera directa por Jean Paul Sastre, escribe un conjunto de obras, entre las cuales se destacan: La invitada (1943), La sangre de otros (1944) y Los mandarines (1954); en ellas, delibera sobre los dilemas de la existencia de la libertad y la responsabilidad individual. En El segundo sexo (1949), muestra que el sexo y la reproducción son el objeto de la pérdida de la libertad. Serret narra que, en esta obra, Beauvoir expone dos tesis que justifican la subordinación: a) que el factor biológico que diferencia al hombre de la mujer, por sus genitales, ha permitido la jerarquización injustificada de un sexo sobre el otro, y b) que“La cercanía de las mujeres con la naturaleza, a partir de la función biológica procreadora, ha generado una imagen universal de mujer-madre-naturaleza, opuesta a la del hombre creador de la cultura, porque, la cultura es superior a la naturaleza." El libro de la francesa, afirma Serret, marca un nuevo giro en la reflexión acerca de la mujer, puesto que" expulsa el discurso feminista fuera del ámbito estricto de la crítica ética, del reclamo por la autonomía, y lo coloca en un terreno más complejo de la realidad, ya no jurídica o moral o civil o incluso política, sino cultural o simbólica" (Serret, 2008, p. 42). El libro de Beauvoir se ha convertido, entonces, en el primer engranaje teórico en criticar la condición biológica, que determina a las mujeres a la sumisión.

Los años sesenta y setenta se caracterizaron por un desencantamiento del poder político, a raíz del fracaso de los movimientos de izquierda y la extensión del capitalismo. Esto provocó un estado de descontento y sospecha, ante cualquier forma de poder. En la nueva atmósfera, aparecen -en escena- las mujeres, los homosexuales, los jóvenes, los desposeídos y todos aquellos afectados por las relaciones de clase; como los nuevos sujetos políticos. De este modo, surge -en la academia- un movimiento feminista denominado "El movimiento por la liberación de la mujer", influenciado por las ideas marxistas y el pensamiento de Beauvoir, quien cuestiona las ideas acerca de la 
sexualidad femenina. El análisis de las investigaciones de ese movimiento, se mueve en tres niveles: a) cuestionar la relación de lo público y lo privado; b) revisar la influencia de lo público en lo doméstico, y c) la casa como objeto de estudio, lugar donde se ejerce el poder (Serret, 2008, p 43-44).

El movimiento por la liberación de la mujer observa la esfera privada, el de la pareja, y coloca en evidencia las relaciones de desigualdad y abuso de poder de un género sobre otro: "la idea de lo doméstico, como el espacio no (muy) visible que permite a lo público, en la modernidad, concebirse como un espacio desigual." Es decir, la casa es el lugar donde el hombre, especialmente, ejerce la violencia psicológica, física y simbólica sobre la mujer, y el espacio donde la mujer está confinada a ejercer las labores domésticas, la maternidad y la crianza de los hijos. La esfera privada, vista de esta manera, es el ejercicio de la libertad masculina, económica y política, a cambio, de la privación de la autonomía femenina (Serret, 2008, p. 45, 46).

Cherry Ortner -antropóloga norteamericana, formada bajo los preceptos del pensamiento de Lévi Strauss, de las investigaciones de Margaret Mead y de las ideas de El segundo sexo, de Simone de Beauvoir- plantea, en su trabajo titulado iEs la mujer respecto al hombre lo que la naturaleza respecto a la cultura?, que las mujeres han estado subordinadas, en todas las sociedades conocidas, por su capacidad biológica para dar a luz y amamantar; por cierto, este es un atributo asociado a la naturaleza, a la belleza y a la función de preservar y cuidar la especie. Dichas características la ponen en desventaja con las funciones masculinas, asociadas a la creación de la cultura. En este sentido, dice la antropóloga, se construye todo un imaginario de segregación que históricamente ha servido para asignar los sitios sociales para cada sexo, atribuyendo -a la mujer- un lugar de desprestigio (Serret, 2008, p. 46).

El aporte de Ortner consistió, dice Serret, en plantear tres hipótesis que recogen las causas de la subordinación de la mujer: a) la biologicista, que consiste en justificar la debilidad, desigualdad social y los roles femeninos, en la condición biológica. Esta hipótesis fue desmontada en los trabajo de la antropóloga, puesto que, en sus investigaciones etnográficas, muestra que hay culturas, cuyas actividades ejercidas por el hombre, como la pesca, son realizadas también por mujeres; b) la historicista, basada especialmente en la tesis de Engles, según la cual la aparición de la propiedad privada, es la causa de la desigualdad y el surgimiento de la dominación masculina. Esta hipótesis fue confrontada por Ortner, en el análisis de los mitos de origen de los pueblos ágrafos, donde imaginan las sociedades matriarcales como pueblos caóticos que requieren la mano del hombre para establecer el orden; esto lo llevó a inferir, una vez más, que el patriarcado siempre ha estado presente en todas las culturas, y, por ende, no tiene una fecha de origen; c) la cultural muestra que la dominación masculina se ejerce a través del lenguaje, que se institucionaliza en las prácticas y discursos sociales, y define la forma de comportamientos, el decir y el pensar de los grupos humanos (Serret, 2008, p. 47-48).

En suma, el sexo y el género son dos conceptos complejos, polisémicos, que han servido para justificar históricamente la represión y dominación de un género sobre el otro. En este aspecto, la labor feminista -desde el análisis histórico, antropológico y etnográfico- ha contribuido a visibilizar la negación de la mujer como un sujeto social, político, histórico y jurídico; y, por su defecto, la creación de políticas públicas, como la perspectiva de género.

\section{La perspectiva de género}

La palabra perspectiva viene del latin perspectious. Significa relativo a lo que se mira: prefijo per: a través, de la raíz specere: mirar, observar y del sufijo tivo: relación activa o pasiva (Diccionario etimológico, 2016). El Diccionario de dudas prehispánicas (RAE, 2005) la concibe como "técnica de representación de la realidad en una superficie plana' o 'punto de vista'"'. Para Ferrater Mora (2004), la perspectiva es la posibilidad de considerar una cosa $y$, en general, el mundo desde diversos puntos de vista; todos ellos justificados. La reunión de perspectivas efectivas y posibles daría la margen verdadera de cada cosa, y solo ella podría ser la verdad absoluta. Así que, para este autor, "un mundo sin perspectivas, es un lugar sin contornos" (Mora, 2004, p. 2767). En este sentido, al observar los fenómenos desde un punto de vista, dotamos y asignamos -a los seres y a las cosas- de existencia, de significado y de sentido. Por ende, la perspectiva de género va 
a constituirse en una nueva forma de ver, con unos lentes que permitirán desvelar lo oculto, lo latente, lo considerado como familiar y cotidiano, en la relación hombre - mujer; pues sin esta perspectiva, el mundo es observado e interpretado a través de una sola mirada: la del hombre.

El término de perspectiva de género hace su aparición -por primera vez, en la escena académica y mundial- en la Cuarta Conferencia sobre la mujer, en Beigin, 1995 (Scott, 2011, p.). La declaración permitió el empoderamiento de la mujer y la creación de políticas públicas internacionales y programas de acción que buscan la igualdad de géneros y la reducción de las formas de discriminación. En ese momento, la perspectiva de género es un conjunto de normas jurídicas, políticas públicas y educativas encaminadas a subsanar la brecha de desigualdad entre los géneros, y prevenir la violencia contra la mujer (Lamas, 1996; Largade, 1996; Gamba, 2005). Sin embargo, el protagonismo de la mujer y las luchas feministas por visibilizar los derechos de las mujeres data del siglo XVIII, proceso histórico imprescindible en la creación de políticas públicas, en las últimas décadas del siglo XX.

La perspectiva de género, para Lamas, juega un papel importante: busca desentrañar los tipos de relación que existen en las ideas construidas simbólicamente por la sociedad. Entre los análisis de la perspectiva de género, se descubre la explotación de la mujer en el mundo laboral, la mala remuneración, la interiorización de modelos de feminidad, a tal punto que las mujeres aceptan -como normales- la forma de estar en el mundo y los modos de violencia. De ahí que Lamas (1995) exprese:

"situación de marginación de las mujeres, la valoración inferior de los trabajos femeninos, la responsabilidad del trabajo doméstico, el constante abandono del mercado de trabajo en años esenciales del ciclo de vida, la insuficiente formación profesional, la introyección de un modelo único de feminidad y el hecho de que, en muchos casos, las mujeres no reconozcan su estatuto de víctimas de la discriminación" (Lamas, 1995, p. 20).

Por su parte, Largarde (1996) piensa que la perspectiva de género es una nueva filosofía posthumanis- ta, surgida de la crítica a la concepción androcéntrica occidental que piensa el universo, la naturaleza y el conocimiento desde el hombre, y deja, a un lado, a las mujeres. De hecho, esta perspectiva, para ella, es un nuevo paradigma histórico-crítico del feminismo; puesto que permite analizar y comprender las características que definen a hombres y mujeres, las posibilidades vitales, el sentido de sus vidas, la complejidad de las relaciones entre ambos géneros, el reconocimiento de los conflictos cotidianos y ampliar la mirada estrecha sobre las relaciones entre géneros. Es más, reconoce que existen carencias, injusticias, pobreza, ignorancia, violencia que invisibilizan a la mujer. Por tanto, significa un reconocimiento de la diversidad de géneros, una nueva resignificación de la historia, de la sociedad, de la política y la cultura (p. 16-17).

Mirar la realidad con perspectiva de género es aspirar a definir las reglas de juego de lo público y lo privado, del valor del éxito, de la sostenibilidad, de las relaciones entre cercanos y extranjeros (Donosso, 2013, p. 75). El desconocimiento de una perspectiva de género conduce a la existencia de graves delitos sexuales y afectaciones, en términos de discriminación de la mujer. Optar por esta perspectiva permite conocer las diferencias y particularidades que se crean entre hombres y mujeres, a partir de la interacción, sus características biológicas, sociales y culturales (A.C.R, 2014, p. 5).

Por otra parte, el uso del término tiene sus desventajas y sus problemas. Primero, la unanimidad social de la naturalización de lo que significa ser hombre y ser mujer está interiorizada en las esferas sociales, de tal manera que se conciben ciertos comportamientos, prácticas, actitudes, deberes y prohibiciones de cada sexo como aspectos innatos en hombres y mujeres. Esta situación hace que se rechace la nueva la perspectiva como un agente perturbador de las prácticas sociales naturalizadas.

La perspectiva de género no es una ideología más, ni un análisis endosable a las concepciones previas. Si somos personas conservadoras, pone en crisis toda nuestra concepción del mundo, nuestros valores, nuestros modos de vida, y la legitimidad del mundo patriarcal. En cambio, si somos mujeres y hombres en transición, democráticos y alternativos, encontramos en esta perspectiva los 
argumentos y los conocimientos para convalidar discrepancias y alternativas, y además para aprender (Lagarde, 1996, p. 21).

Segundo, la mirada estrecha de quienes conciben género -como sinónimo de mujer, desconociendo que género hace alusión a los dos géneros (ACR, 2014) - neutraliza los análisis, la comprensión y la crítica de los estudios sobre género. Tercero, el desgaste del término, en la formulación de políticas públicas, convierte la perspectiva de género en víctima de la burocratización, por parte de quienes realizan acciones de orden social. (Largarde, 1996). Sumado a la anterior situación, existe el desconocimiento de las comunidades universitarias sobre el tema, ya que no existen programas ni currículos ni docentes capacitados para abordar las problemáticas acerca de la perspectiva de género (Donosso, 2013, p. 73-74). También se consideran los planteamientos sobre género como subversivos, porque, dizque, atacan la moral de la sociedad y los valores familiares, al sustentar que puede haber más de un género (Scott, 2011). Consecuencia de tales situaciones son las reacciones negativas contra los movimientos feministas que promulgan sus estudios.

Así pues, adoptar una perspectiva de género implica; primero, reconocer relaciones desiguales y discriminatorias entre géneros, que histórica y socialmente están inmersas en los individuos y en el entramado social, como las de clase, etnia, edad, preferencia sexual y religión. Segundo, declarar que la discriminación se expresa en todos los ámbitos de la cultura: el trabajo, la familia, la política, las organizaciones, el arte, las empresas, la salud, la ciencia, la sexualidad, la historia. Y, tercero, aceptar que la perspectiva de género posibilita una nueva reconstrucción epistemológica de la realidad, desde los diversos géneros (Gamba, 2008).

Para comprender mejor los objetivos y alcances de la perspectiva de género, es importante acercarse a algunas definiciones del concepto categoría de género, pues este se ha constituido en una categoría analítica para comprender las formas de discriminación hacia la mujer, encubiertas en todos los ámbitos sociales y en las diferentes expresiones culturales. Por tal razón, es indispensable realizar un recorrido por definiciones de los términos sexo y género.

\section{El concepto de sexo}

Gayle Rubín (citado por Serret, 2008; Barbieri, 1993) es la primera en plantear que el sexo y el género son un solo sistema. Para Rubín, el sexo es la condición biológica con que los seres humanos nacen y se determina por los órganos genitales. A partir de esta diferenciación natural, la sociedad construyó un conjunto de productos que transformaron la misma sexualidad, y se atribuyeron nombres, etiquetas, status, jerarquías, formas de actuar, de comportamiento y de pensar. A este conjunto de cualidades incorporadas socialmente al sexo, Ruby la denomina género.

El sexo determina el destino de los sujetos femeninos o masculinos en las sociedades humanas. Se había concebido, usualmente, que nacer con los genitales masculinos proporcionaba al sujeto una gama de posibilidades, status y privilegios mayores que no tienen las mujeres. Nacer mujer posibilita al sujeto femenino el poder de ser madre y la capacidad de reproducirse que no la tienen los hombres; sin embargo, estos atributos la condenan a una determinada forma de ser y actuar, vigilada socialmente. Idea que fue reforzada por biólogos del siglo XIX, como Brooks, Geddes y Thomas, quienes llegaron a afirmar que los órganos genitales determinaban la personalidad, las capacidades intelectuales de los individuos y su identidad. Tesis que fue refutada por las investigaciones de las feministas del siglo $X X$ (Castellanos, 2007). Para Castellanos, no es lo biológico lo que determina la personalidad de un sujeto, como pensaron los biólogos, sino las costumbres, las formas de actuar, pensar y decir; trasmitidas por la sociedad y que moldean las concepciones hacia lo femenino o masculino.

Entre otras definiciones del sexo se pueden destacar las siguientes:

- Kate Miller, en su obra La política sexual, considera que el sexo tiene dimensiones políticas desconocidas que se manifiestan en las relaciones diarias entre los individuos; no es lo mismo ser hombre que ser mujer en sociedades no democráticas.

- Para las feministas influenciadas por Foucault, el sexo es producto de los discursos y prácticas sociales inculcadas en los diferentes contextos culturales, que meramente un resultado de la naturaleza. 
- La antropóloga feminista Henrietta Moore argumenta que los cuerpos no tienen sexo por fuera de los discursos construidos socialmente, pues son los discursos mismos los que refuerzan e institucionalizan la idea de sexo. Demuestra su afirmación, a partir de dos estudios etnográficos que realiza en Nepal y en el pueblo Hua, de Nueva Guinea. En las culturas de Nepal, encontró que los cuerpos son una combinación de lo femenino y lo masculino, es decir, el cuerpo es visto como una aleación entre lo femenino, que es la carne, y lo masculino, que son los huesos. Ella dice que, en este pueblo, los sujetos no tienen sexo, puesto que este depende de la edad y de los líquidos corporales que determinan la forma de actuar en los diferentes momentos de la vida. En esta cultura, la ideología heterosexual no es una marca exclusiva, ni tampoco es sancionada socialmente. Una mujer curandera puede asumir actitudes masculinas, $\mathrm{O}$ femeninas.

- Los estudios Miller y Moore contribuyeron a desvirtuar el determinismo biológico del sexo, aceptado de manera general por la sociedad; ellos conciben el sexo como producto cultural (Castellanos, 2007, p. 224-227).

- Los estudios etnográficos de Gloria Wekker y Mary Ann Clark corroboran la afirmación de Moore, puesto que, para ellas, el sexo es un producto de la cultura. Por su parte, Wekker realizó un estudio en los grupos de la religión afro-surinamesa y encontró que los integrantes de los Winti no separan, de manera radical, lo masculino de lo femenino. Creen que las personas están constituidas por la personalidad de los dyodyo (padres del mundo de los dioses) y un kra, un alma inmortal; dicen que tanto el uno como el otro están compuestos por ingredientes femeninos y masculinos. Los Mati, otro grupo de esa religión, no creen en una orientación sexual innata. Las líderes espirituales que actúan también como sacerdotisas y parteras, aunque tienen esposo, tienen relaciones sexuales con mujeres; sin embargo, no son consideradas como lesbianas o bisexuales. Pues piensan que son guiadas por un espíritu masculino -Apukuquien se siente atraído por las mujeres.

- Los estudios de Mary Clark fueron realizados con los grupos Santeros de Cuba, los Orichas. La investigadora encontró que los santeros -independientemente de su sexo, género u orientación sexual- presentan comportamientos masculinos o femeninos, especialmente, los roles de este último. Los Orichas, igual que sus dioses, pueden ser masculinos o femeninos, pues ambas fuerzas intervienen en la creación del universo. Changó, dios del trueno, es representado por una virgen, Santa Barbara, a quien se le puede rezar como papá o virgencita santa. Evidentemente, en la cosmovisión de la santería -argumenta Gabriela Castellanos- no se encuentra "la división tajante e inescapable entre los dos sexos a la que estamos acostumbrados en la tradición judeo-cristiana" (p. 228).

- Thomas Laqueur, citado por Castellanos, demuestra en sus trabajos que las concepciones acerca del sexo varían de acuerdo con el momento histórico, cultural y político de cada sociedad. Este autor encontró que, en la Antigüedad Clásica, se presentaba el sexo como único: hombres y mujeres tenían el mismo sexo. La diferencia estaba en el desarrollo de sus genitales, pues se consideraba a la mujer con genitales atrofiados. Esta idea dio pie a creer que el hombre es superior a las mujeres (Castellanos, 2008, pp. 224-229).

De esta manera, los estudios realizados por los investigadores mencionados dan cuenta de que el sexo es la condición biológica con que los seres humanos nacen determinados por los genitales, pero, al igual que el género, es una construcción simbólica sobre los cuerpos. Y, desde esa óptica, se produce un conjunto de ideas enseñadas y trasmitidas socialmente, que justifican la clasificación, el sexismo, los comportamientos, las actitudes, las prácticas sociales y la violencia; entre sexos.

\section{El concepto de género}

De acuerdo con Castellanos (2007), el primer registro histórico del término género (Gender) data del siglo XVII, usado por la inglesa Lady Mary Wortley Montagu, quien fue la primera persona que lo usó (gender), refiriéndose, de manera despectiva, a su propio sexo. Ella señalaba que"lo único positivo de pertenecer a ese grupo es la seguridad de no casarse con ninguno de sus miembros". En Francia, se empezó a usar desde la época victoriana (1837-1901), como eufemismo, pues indicaba la diferencia física entre hombres y mujeres: era de mal gusto emplear el término sex. Paulatinamente, de la diferencia física 
se pasó a señalar los estilos y comportamientos entre hombres y mujeres.

Castellanos (2007) plantea que la palabra "género" se familiarizó y se extendió hasta ser aceptada y reconocida por la academia norteamericana de Ciencias Sociales del siglo XX. En 1955, el sociólogo Jhon Mooney propuso la expresión "gender roles", para mostrar la conducta esperada de hombres y mujeres. En 1968, el psicoanalista Robert Stoller, en su obra Sexo y género, argumenta la tesis de la distinción entre masculinidad y la feminidad, a partir de la diferencia biológica. En 1975, la antropóloga Gayle Rubien, en su artículo "El tráfico de mujeres", realiza la primera definición del sistema sexo-género. En 1986, la historiadora Jhon Scott, en su obra El género: una categoría útil para el análisis histórico, define el género como"un elemento constitutivo de las relaciones sociales que se basa en las diferencias entre sexos" " $^{\text {"una forma }}$ primaria de las relaciones de poder". La definición de Scott, dice Castellanos, presenta tres elementos que la integran: a) sin el género, no es posible hablar de masculino y de femenino, ni de la existencia del uno y del otro; la sociedad está construida a partir de relaciones madre-padre, hermano-hermana, las cuales, desde la infancia, se van interiorizando; b) se dimensiona la esfera política que subyace en el concepto poder. Este, no necesariamente, es la división política y jerárquica de clases sociales, sino que el "poder se maneja en los discursos: quienes definen los términos y quienes lo emplean están involucrados en el juego de poder"; parafraseando a Castellanos, el poder se institucionaliza, circula, se transmite en los discursos, se reproduce entre los mismo sujetos; especialmente, en las relaciones de familia. Su objetivo es controlar, vigilar y penalizar la sexualidad. El padre repite los esquemas sociales y se convierte en un agente de dominación; por su parte, la madre, las tías, las abuelas se convierten en sujetos de dominación también, al controlar la sexualidad de los hijos; c) el género es el conjunto de saberes sobre los que descansan las diferencias de los sexos realizadas por la cultura.

Los saberes se reproducen y se comparten a través de determinados tipos de discurso, desde los científicos hasta los narrativos tanto los relatos literarios como los de la vida cotidiana, pasando por toda la gama de discursos profesionales, más o menos especializados. Es allí, en lo que la gente dice y escribe, donde se juegan las batallas que se decidirán lo que consideramos verdad, lo que consideramos legítimo, lo que consideramos valioso e importante. Es allí donde se establecerá quien tiene derecho a tomar determinadas decisiones en la vida social, es decir, quien ostentará cada tipo de poder. (Castellanos, 2007, p. 232).

En este orden de ideas, el género es un conjunto de saberes y discursos que se reproducen en las disciplinas y en las relaciones intersubjetivas, en el hablar, en la escritura, en el decir; así, se convierte en verdad. Sobre este asunto, Castellanos afirma:

Este conjunto de saberes, prácticas y relaciones de poder configuran los cuerpos, la sexualidad, las diferencias físicas, económicas y políticas entre sexos en una época y contexto determinado. Es decir, que el género, no solo es la construcción que se hace del sexo, sino también de la sexualidad y las diferencias e identidades de los sexos. (Castellanos, 2007, pp. 236-237).

\section{El generoelecto}

Cabe considerar, por otra parte, que sentirse hombre o mujer depende, entre muchos otros factores, del lenguaje y de lo que Deborath Tannen ha denominado estilo de género o géneroelectos. Los seres humanos incorporan formas de actuar y de ser a través del lenguaje, puesto que, a medida de que se interactúa y se habla, se está construyendo la identidad. A las distintas formas de ser mujer y de ser hombre reforzadas por el lenguaje, es lo que Butler denomina actos performativos (expresión empleada en el texto Gender Trouble, 1990). Los sujetos femeninos o masculinos confirman su identidad cada vez que hablan; con ello, afirman lo que son: "Yo soy mujer", "yo soy hombre”, acompañado de actos corporales (Castellanos, 2007).

Hoy, en tiempo de mayor tolerancia, es posible decir: "soy homosexual", "o "soy gay". Estas expresiones eran inconcebibles en el mundo décadas atrás, pues la heterosexualidad ha sido un factor dominante en nuestras sociedades. En la actualidad, gracias a las deliberaciones, reflexiones y debates sobre los derechos a la igualdad y a la diferencia, muchos hombres y mujeres manifiestan su identidad sexual. 
Cada uno de nosotros llega a identificarse como hombre o como mujer al realizar actos de lenguaje mediante los cuales nos designamos directa o indirectamente, como pertenecientes a uno u otro sexo. Por otra parte, los mismos actos corporales que realizamos, las formas de movernos y gestualizar que de algún modo aprendemos como"generalizados", es decir, correspondientes a uno u otro género, van conformando un estilo que contribuye a moldear nuestra identidad (Castellanos, 2007, p. 239).

Al respecto, Judith Butler, dice Lamas señala que el género es un hacer que está conformado por el sexo, por el deseo sexual y por la práctica social; y se deriva en actos performativos; es decir, "cómo una actuación coercitiva y ficticia se presta a un acto subversivo" (Butler, citado por Lamas, 2000, pp. 8). Para Butler "el género es el producto, mediante el cual las personas reciben significados de la cultura, pero también está dispuesto a innovar. En otras palabras, el ser humano incorpora prácticas y representaciones sociales y actúa en lo que quiere ser. No obstante, Butler, agrega Lamas, se atreve a lanzar la idea provocadora de que el "género" es un proyecto para renovar la historia cultural, que se hace de manera voluntaria y luego se arraiga en la cultura (Lamas, 2000, 7-8). "Nos encontramos ante el montaje físico-psico-sociológico de una serie de actos, más o menos habituales (habitus) y más o menos viejos en la vida del hombre y en la historia de la sociedad (Mauss, citado por Lamas, 2000, p. 9). De ahí que el ser humano asume ciertas formas de actuar, pero está en las condiciones de transformar voluntariamente el destino; por tal motivo, se convierte en un acto de constante rebelión.

Para Lamas no basta la concepción de género, como performativo, como actuación. La emancipación significa decodificar significados y metáforas estereotipadas, cuestionar las ficciones regulativas, criticar la tradición y las resignificaciones paradójicas. Es comprender también que la diferencia entre los sexos está basada en el conjunto de oposiciones construidas a partir de la diferencia anatómica, que luego se interioriza en "esquemas de pensamiento", y se registran como diferencias naturales; de esta manera, se convierten en habitus, en costumbres. En la oposición de lo femenino y lo masculino, aprendido en las prácticas cotidianas, es donde las personas estructuran la percepción y la organización simbólica de la vida social (Lamas, 2000). La oposición entre las sexos se convierte en algo tan natural que se asume sin justificación y se convierte en algo casi evidente,

Estas estructuras cognitivas se traducen en esquemas no pensados de pensamiento en habitus mediante el mecanismo básico y universal de la oposición binaria, en forma de pares: alto/bajo, grande/pequeño, afuera/adentro, recto/torcido, etc. Estos habitus son producto de la encarnación de la relación de poder, que lleva a conceptualizar la relación de dominación/dominado como natural (Lamas, 2000, p. 11).

Por otra parte, las diferencias, las formas de dominación, el lenguaje, la crianza, las normas, los valores, que proceden de la cultura, se anclan en los cuerpos, se inscriben en lo biológico, en el subconsciente. En palabras de Lamas, los cuerpos pasan por una construcción biologizada, que se convierte en un habitus (Lamas, 2000). El cuerpo, para Lamas, es "como un ente/artefacto, simultáneamente físico y simbólico, producido tanto natural como culturalmente, y situado en momento histórico concreto y en una cultura determinada. El cuerpo experimenta, en el sentido fenomenológico, distintas sensaciones, placeres, dolores, y la sociedad le impone acuerdos y prácticas psicolegales y coertivas." (Lamas, 2000, p. 12) Esto es, para el caso de la mujer, la incorporación en su cuerpo y mente de formas de comportarse, de pensar, de cuidar y criar los hijos, de ser pudorosa, de participar en las labores domésticas. En otras palabras, al cuerpo de la hembra se le impone la feminidad y al cuerpo de los machos, la masculinidad.

Según Deborath Tannen (1993), los generoelectos representan el juego de oposiciones, entre géneros y prácticas interiorizadas culturalmente en los cuerpos de hombres y mujeres, más el factor natural. Esta autora analiza las diferencias de "género" en la construcción comunicativa, y encuentra que los varones y las mujeres se comportan de acuerdo con estereotipos. Por ejemplo, afirma que el generoelecto masculino se caracteriza por: tener relaciones jerárquicas con el mundo, luchar incesantemente por el espacio personal, valorar el éxito personal y la autonomía; buscar la superioridad, la competencia, el desafío, las disputas verbales; temer el fracaso, evi- 
tar solicitar información, pues considera que pedirla es cuestión de debilidad, por eso, decide guardar silencio y no solicitar ayuda. A cambio de esto último, elige la lucha personal, la confrontación directa, ya sea mediante el uso de la palabra, o la confrontación física.

En cambio, el generoelecto femenino es todo lo contrario al masculino: valora las relaciones interpersonales, establece lazos fuertes y duraderos, busca expresar sus sentimientos personales. La debilidad, más que ser una desventaja, es una estrategia poderosa para que el otro se "ponga a sus pies"y la comprenda, con el fin de no perder la relación afectiva. Teme la soledad, no tener quien la escuche o la pueda consolar. No teme pedir información, pues le permite conocer personas y salir de problemas. En los conflictos, busca el consenso, la conciliación, el disimulo, porque le interesan los lazos afectivos más que el rompimiento y el aislamiento de las relaciones sociales. Teme no ser querida, valora la igualdad y la simetría, más que la autonomía. De este modo, para Tannen, hay formas diferentes de actuar de los varones y mujeres en la sociedad, que se agrupan en lo que ha denominado generoelecto. Por ejemplo, un hombre, con generoelecto masculino, puede tener algunos rasgos del "generoelecto" femenino. No necesariamente puede buscar la confrontación, sino apelar a la conciliación o a la simetría. O puede haber mujeres con generoelecto femenino que temen el fracaso e intentan imponerse sobre los demás, desafiando a su interlocutor, mediante una contienda verbal. También, puede haber mujeres competitivas que valoran la autonomía, más que las relaciones simétricas e igualitarias. Los generoelectos no son categorías, sino que evolucionan según la historia y el grupo étnico al cual pertenecen (Deborath, citada por Castellanos, 2007, p. 240-244).

Con todo lo anterior, puede decirse que las formas de ser hombre o mujer son el resultado de una construcción biologizada de las normas sociales, valores, prácticas, costumbres, hábitos, roles que se interiorización en el cuerpo y en las estructuras mentales de las personas; expresadas y matizadas en el momento de hablar y actuar. Las estructuras mentales conformadas pueden tipificarse en lo que se denomina generoelecto, que puede variar según el contexto, la cultura, y el momento histórico.

\section{Conclusión}

A manera de reflexión, es posible afirmar que:

- Los estudios de género y el enfoque de perspectiva de género han significado un gran avance en la investigación en las ciencias sociales, pues han permitido aclarar las formas desiguales y discriminatorias entre géneros, interiorizadas históricamente en las instituciones sociales y en todo el entramado social como: la clase, la etnia, la edad, la religión, la familia, el trabajo, las ciencias, las artes, etc. Las políticas públicas, en pro de la defensa de los derechos de la mujer, es un resultado de estos estudios. Es la invisibilidad ante el ostracismo de la que fue víctima, por muchos siglos, a causa de las ideas preconcebidas culturalmente de lo que significa ser mujer. Estas ideas minimizaron las capacidades de la mujer y la han ubicado en un grupo reducido de labores y oficios, en la esfera individual y pública.

- Estos estudios muestran que el sexo y el género son dos categorías distintas y complejas, determinadas por la cultura en la cual vive el individuo. Pero lo dicho muestra que el género es la construcción simbólica realizada por la cultura sobre los cuerpos, especialmente, sobre el sexo, o la condición biológica, en la cual se nace. Este constructo se da desde los saberes, los discursos, las prácticas, las formas de hablar, de escribir, de decir, en las formas de comportarse, de relacionarse: así, se constituyeron las formas de masculinidad o feminidad. Y, en consecuencia, ha servido para justificar el sexismo, la discriminación, las relaciones de poder, la desigualdad entre géneros, y la exclusión de aquellos que se no identifican con la heterosexualidad.

- Es un hecho que el mundo ha evolucionado en materia de derechos, pero el grado de tolerancia hacia la diferencia ha aumentado, paulatinamente. Sin embargo, las nuevas generaciones luchan por construir una identidad, para ser aceptados y reconocidos, frente a la sociedad de consumo, de familias disfuncionales o la inexistencia de la misma, de la falta de la solidaridad, de la ausencia de ideales colectivos de una nación, de los conflictos armados, de la pérdida de valores, de las actitudes de discriminación de menosprecio frente a la diferencia.

- Los estudios de género, con la aclaración de la categoría de sexo y género, han posibilitado, en las 
últimas décadas, cambiar la mirada hacia la mujer y hacia los otros géneros.

\section{Referencias}

Agencia Colombiana para la Reintegración. (2014). Perspectiva de género en el proceso de Reintegración. Estrategia de Género de la ARC: Implementando un enfoque diferencial basado en la consideración del individuo y sus caracteristicas. Agencia Colombia para la reintegración. (Eds.). Bogotá, Colombia. 17 p.http://wwww.reintegracion.gov.co/es/la-reintegracion/centro-de-documentacion/Documentos/Perspectiva\%20de\%20 g\%C3\%A9nero\%20en $\% 20$ el $\% 20$ Proceso $\% 20$ de\%20Reintegraci\%C3\%B3n.pdf.

Castellanos- Llanos, G. (2007). Sexo, género y feminismo: tres categorias en pugna. Revista Género, 8(1), 223-251. http://www.revistagenero.uff. br/index.php/revistagenero/article/view/168/109.

Deleuze, G., \& Guattari, P. F. (1999). ¿Qué es la filosofía? Anagrama (Eds.). Colecciòn: Argumentos. Barcelona, España. 224 p.

Diccionario Etimológico. (2016). http://etimologias.dechile.net/?perspectiva.

Donoso-Vásquez, T., \&Velasco- Martínez, A. (2013). ¿Por qué una propuesta de formación de género en el ámbito Universitario? Universidad de Barcelona (Eds.) Revista de Currriculo y formación del profesorado, 17(1), 71-88. http://wwww. ugr.es/ recfpro/rev171ART5.pdf.
Gamba, S. (2008). Agenda de las mujeres. El portal de las mujeres Argentinas, Iberoamericanas y del Mercosur. http://agendadelasmujeres.com.ar/ index2.php?id=3Enota $=5703$.

Lagarde, M. (1996). Género y feminismo. Desarrollo Humano y Democracia. Horas y Horas (Eds.) Madrid, España. 200 p.

Lamas, M. (2011). La tarea. Revista de Educación y Cultura, de la sección 47 del SNTE. 8. http:// www.latarea.com.mx/articu/articu8/lamas8.htm.

Lamas, M. (2000). Diferencia de sexo, género y diferencia sexual. Cuicuilco, 7(18). http://www.redalyc.org/articulo.oa?id=35101807.

Mora, F. (2004). Diccionario de Filosofía (Vol. 1). Ariel S. A. (Eds.). Barcelona, España.

Real Académia Española. (2005). Diccionario de prehispanico de dudas. http://lema.rae.es/dp$d /$ ?key=perspectiva .

Reinhart, K. (2007). Historia e historia. Norba. Revista de Historia, 20, 209-224.

Scott, J. (2011). El género: ¿Todavía una categoría útil para el análisis? La Manzana de la discordia, 6(1), 95-101. http://www.bdigital.unal.edu. co/48429/1/g\%C3\%A9nerotodav\%C3\%ADaunacategor\%C3\%ADa.traducci\%C3\%B3n.pdf.

Serret-Bravo, E. (2008). Qué es y para qué es la perspectiva de género. Libro de texto para la asignatura: Perspectiva de Género en la Educación Superior. Instituto de la Mujer Oaxaqueña (Eds.). Oaxaca, México. 158 p.

http://equidad.pueg.unam.mx/sites/default/files/files/ oax09.pdf. 